\title{
THE ALGEBRA OF BESSEL SEQUENCES AND MEANS OF FRAMES IN HILBERT SPACES
}

\author{
ESMAEIL ALIZADEH, ASGHAR RAHIMI, AND MORTAZA RAHMANI
}

Received 11 October, 2019

\begin{abstract}
In this paper, a one-to-one correspondence between Bessel sequences and bounded linear operators is provided. This leads to an algebra structure on the set of all Bessel sequences in a separable Hilbert space. Some kinds of frames as special classes of operators are considered. Also, normal Bessel sequences and positive frames are presented. Finally, power means of positive frames are introduced. These allow researches to construct a large number of new frames from existing frames.
\end{abstract}

2010 Mathematics Subject Classification: 42C15; 41A58; 42C40

Keywords: Bessel sequence, positive frame, frame operator, means of frames

\section{INTRODUCTION AND PRELIMINARIES}

Frames in Hilbert spaces were introduced by Duffin and Schaeffer [5] as a part of their study in non-harmonic Fourier series. Frames did not find serious attention until 1986 when Daubechies, Grossmann and Meyer [2] brought it all back to life during their fundamental work on wavelets.

There are many generalizations of frame. Most of these generalizations have been found useful applications. Some generalizations of frame significance have been presented such as generalized frames [12,13], continuous frames [7], continuous fusion frames [6] and K-frames [8]. In the sequel, we discuss results on one such generalization of frames, called K-frames.

In recent years many researchers studied some methods for constructing new frames from existing ones. In 2009, Obeidat et al. [11] studied sum of two frames.

A basic and frequently used tool in study and characterizations of frames is the frame operator. It is known that the frame operator of a given frame is a bounded positive invertible operator. Since two different frames may have the same frame operator, a natural question arises here: Is there a way to correspond each Bessel sequence to one and only one bounded linear operator such that its properties characterizes special kinds of frames? 
In this paper, we find an appropriate correspondence between Bessel sequences (some kinds of frames) with bounded linear operators. Using this relation, we introduce an algebraic structure of the set of Bessel sequences in Hilbert spaces. Also, we study the means of frames.

Now, we recall some basic definitions and results that we need in the sequel. For background material on frame theory and the related topics, we refer to [1]. Throughout the paper $\mathcal{H}$ is a separable Hilbert space with an arbitrary fixed orthonormal basis $\left\{e_{j}\right\}_{j \in J}$ where $J \subseteq \mathbb{N}$. We denote by $B(\mathcal{H})$ the space of all bounded linear operators on $\mathcal{H}$. For $A \in \mathcal{H}$, we denote $R(A)$ for range of $A$. A bounded operator $A \in B(\mathcal{H})$ is called positive if $\langle A f, f\rangle \geq 0$ for all $f \in \mathcal{H}$. Let $B_{+}(\mathcal{H})$ denote the set of all bounded and positive operators on $\mathcal{H}$. Every positive operator is clearly self-adjoint. For $A, B \in B(\mathcal{H})$, we write $A \leq B$ if $B-A$ is positive. If $A \in B(\mathcal{H})$ is positive, then there exists a unique positive operator $B$ such that $B^{2}=A$. This will be denoted by $B=A^{\frac{1}{2}}$. Moreover, if an operator $C$ commutes with $A$, then $C$ commutes with every operator in the $C^{*}$-algebra generated by $A$ and $I$, specially $C$ commutes with $A^{\frac{1}{2}}$.

Definition 1. A family $F=\left\{f_{j}\right\}_{j \in J}$ of elements in $\mathcal{H}$ is called a Bessel sequence if there exists a constant $\mu>0$ such that

$$
\sum_{j \in J}\left|\left\langle f, f_{j}\right\rangle\right|^{2} \leq \mu\|f\|^{2}, \quad \forall f \in \mathcal{H} .
$$

For a Bessel sequence $\left\{f_{j}\right\}_{j \in J}$, the operator $T_{F}: \ell_{2} \rightarrow \mathcal{H}$ defined by $T_{F}\left(\left\{c_{j}\right\}_{j \in J}\right)=$ $\sum_{j \in J} c_{j} f_{j}$, is bounded. The operator $T_{F}$ is called the synthesis operator (or, pre-frame operator). The adjoint of $T_{F}, T_{F}^{*}: \mathcal{H} \rightarrow \ell_{2}$ defined by $T_{F}^{*} f=\left\{\left\langle f, f_{j}\right\rangle\right\}_{j \in J}$ is called the analysis operator. By composing $T_{F}$ and $T_{F}^{*}$, we obtain the frame operator

$$
S_{F} f=T_{F} T_{F}^{*} f=\sum_{j \in J}\left\langle f, f_{j}\right\rangle f_{j} \quad \forall f \in \mathcal{H} .
$$

Moreover, for each $f \in \mathcal{H}$,

$$
\left\langle S_{F} f, f\right\rangle=\sum_{j \in J}\left|\left\langle f, f_{j}\right\rangle\right|^{2} .
$$

Definition 2. The Bessel sequence $F=\left\{f_{j}\right\}_{j \in J}$ is a frame for $\mathcal{H}$ if there exists a constant $\lambda>0$ such that

$$
\lambda\|f\|^{2} \leq \sum_{j \in J}\left|\left\langle f, f_{j}\right\rangle\right|^{2}, \quad \forall f \in \mathcal{H} .
$$

The constant $\lambda$ is called the lower frame bound for the frame $\left\{f_{j}\right\}_{j \in J}$. Note that this bound is not unique.

For a given frame $F=\left\{f_{j}\right\}_{j \in J}$, the operator $S_{F}$ is a bounded positive self-adjoint operator and hence $S_{F}$ has a unique positive square root, denoted by $S_{F}^{1 / 2}$. The frame operator $S_{F}$ of a given frame is invertible and, in fact, satisfies $\lambda I \leq S_{F} \leq \mu I$. For 
normalized tight frames (that is, for frames with $\lambda=\mu=1$ ), the frame operator is the identity operator.

The notion of $K$-frames has been introduced by Gavruta to study the atomic systems with respect to a bounded linear operator $K$ in a separable Hilbert space. $K$ frames are more general than ordinary frames in the sense that the lower frame bound only holds for the elements in the range of $K$.

Definition 3 ([8]). Let $K \in B(\mathcal{H})$. A sequence $F=\left\{f_{j}\right\}_{j \in J}$ in $\mathcal{H}$ is called a $K$ frame for $\mathcal{H}$ if there exist constants $\lambda, \mu>0$ such that

$$
\lambda\left\|K^{*} f\right\|^{2} \leq \sum_{j \in J}\left|\left\langle f, f_{j}\right\rangle\right|^{2} \leq \mu\|f\|^{2} \quad \forall f \in \mathcal{H} .
$$

Definition 4 ([3]). Let $K \in B(\mathcal{H})$. A sequence $\left\{f_{j}\right\}_{j \in J}$ in $\mathcal{H}$ is said a tight $K$-frame with bound $\lambda$ if

$$
\lambda\left\|K^{*} f\right\|^{2}=\sum_{j \in J}\left|\left\langle f, f_{j}\right\rangle\right|^{2}, \quad \forall f \in \mathcal{H} .
$$

If $\lambda=1$ in (1.2), then the sequence $\left\{f_{j}\right\}_{j \in J}$ is called Parseval $K$-frame for $\mathcal{H}$.

Theorem 1 ([13]). Let $\left\{f_{j}\right\}_{j \in J}$ be a Bessel sequence in $\mathcal{H}$ and $K \in B(\mathcal{H})$. Then $F=\left\{f_{j}\right\}_{j \in J}$ is a $K$-frame for $\mathcal{H}$ if and only if there exists $\alpha>0$ such that $S \geq \alpha K K^{*}$, where $S$ is the frame operator for $\left\{f_{j}\right\}_{j \in J}$.

In the sequel we need the following theorem, which is known as Douglas' factorization theorem.

Theorem 2 ([4]). Let $\mathcal{H}$ be a Hilbert spaces and $A, B \in B(\mathcal{H})$. Then the following conditions are equivalent.

(1) $R(A) \subseteq R(B)$.

(2) $A A^{*} \leq \alpha^{2} B B^{*}$ for some $\alpha>0$.

(3) There exists $X \in B(\mathcal{H})$ such that $A=B X$.

\section{Bessel ALgebra $\operatorname{Bess}(\mathcal{H})$}

We seek a way to express each Bessel sequence (or frame) by one and only one operator. One natural way in this direction is using the frame operator.

Remark 1. Let $\left\{e_{j}\right\}_{j \in J}$ be an arbitrary orthonormal basis for $\mathcal{H}$ and $U \in B_{+}(\mathcal{H})$. It is easily seen that $F=\left\{f_{j}\right\}_{j \in J}=\left\{U^{1 / 2} e_{j}\right\}_{j \in J}$ is a tight $K$-frame with $K=U^{1 / 2}$ for $\mathcal{H}$. Moreover, considering (1.1), for each $f \in \mathcal{H}$, we have

$$
\begin{aligned}
S_{F} f & =\sum_{j \in J}\left\langle f, f_{j}\right\rangle f_{j}=\sum_{j \in J}\left\langle f, U^{1 / 2} e_{j}\right\rangle U^{1 / 2} e_{j} \\
& =U^{1 / 2}\left(\sum_{j \in J}\left\langle U^{1 / 2} f, e_{j}\right\rangle e_{j}\right)=U^{1 / 2}\left(U^{1 / 2} f\right) \\
& =U f .
\end{aligned}
$$


That is the frame operator of the frame $\left\{U^{1 / 2} e_{j}\right\}_{j \in J}$ is $S_{F}$.

The following example shows that two different Bessel sequences (or frames) may have the same frame operator.

Example 1. Let $\left\{e_{j}\right\}_{j \in J}$ be an arbitrary orthonormal basis for $\mathcal{H}$. Define

$$
f_{1}=e_{1}, f_{2}=e_{1}-e_{2}, f_{3}=e_{1}+e_{2}, f_{j}=e_{j-1}(j \geq 4) .
$$

It is easy task to show that $\left\{f_{j}\right\}_{j \in J}$ is a frame with the frame operator $S_{F}$ defined by

$$
S_{F} e_{1}=3 e_{1}, S_{F} e_{2}=2 e_{2}, S_{F} e_{j}=e_{j}(j \geq 3) .
$$

Putting $g_{j}=S_{F}^{1 / 2} e_{j}$, we have

$$
g_{1}=\sqrt{3} e_{1}, \quad g_{2}=\sqrt{2} e_{2}, g_{j}=e_{j}(j \geq 3) .
$$

It follows from Remark 1 that the frame operator of $\left\{g_{j}\right\}_{j \in J}$ is also $S_{F}$.

This example shows that the frame operator is not appropriate for our goal. In the following theorem, we correspond each Bessel sequence to one and only one operator. We denote by $\operatorname{Bess}(\mathcal{H}), \operatorname{Fr}(\mathcal{H})$ and $\operatorname{KFr}(\mathcal{H})$ the set of all Bessel sequences, frames and $K$-frames in $\mathcal{H}$, respectively.

Theorem 3. Let $\left\{e_{j}\right\}_{j \in J}$ be an arbitrary orthonormal basis for $\mathcal{H}$. The Bessel sequences for $\mathcal{H}$ are precisely the families $\left\{U e_{j}\right\}_{j \in J}$, where $U$ is a bounded operator on $\mathcal{H}$. Consequently, there is a one-to-one correspondence between the elements of $B(\mathcal{H})$ and $\operatorname{Bess}(\mathcal{H})$.

Proof. Let $\left\{\delta_{j}\right\}_{j \in J}$ be the canonical basis for $\ell^{2}(\mathbb{N})$ and $\left\{e_{j}\right\}_{j \in J}$ be an ortonormal basis for $\mathcal{H}$. Let $\Gamma: \mathcal{H} \rightarrow \ell^{2}(\mathbb{N})$ be the isometric isomorphism defined by $\Gamma e_{j}=\delta_{j}$. If $F=\left\{f_{j}\right\}_{j \in J}$ is a Bessel sequence, then the pre-frame operator $T_{F}$ is bounded and $T_{F} \boldsymbol{\delta}_{j}=f_{j}$. Putting $U_{F}=T_{F} \Gamma$, we have

$$
\left\{f_{j}\right\}_{j \in J}=\left\{U_{F} e_{j}\right\}_{j \in J}
$$

and $U_{F}$ is bounded. If $U^{\prime} \in B(\mathcal{H})$ is another operator such that $\left\{f_{j}\right\}_{j \in J}=\left\{U^{\prime} e_{j}\right\}_{j \in J}$, then clearly $U^{\prime}=U_{F}$. Therefore, $U_{F}$ is unique.

On the other hand, if $U$ is a bounded operator on $\mathcal{H}$, then for each $f \in \mathcal{H}$ we have

$$
\sum_{j \in J}\left|\left\langle f, U e_{j}\right\rangle\right|^{2}=\sum_{j \in J}\left|\left\langle U^{*} f, e_{j}\right\rangle\right|^{2}=\left\|U^{*} f\right\|^{2} \leq\left\|U^{*}\right\|^{2}\|f\|^{2} .
$$

Hence $\left\{U e_{j}\right\}_{j \in J}$ is a Bessel sequence for $\mathcal{H}$. Moreover, if $S$ is the frame operator of $\left\{U e_{j}\right\}_{j \in J}$, then for each $f \in \mathcal{H}$ we have

$$
\begin{aligned}
S f & =\sum_{j \in J}\left\langle f, U e_{j}\right\rangle U e_{j}=\sum_{j \in J}\left\langle U^{*} f, e_{j}\right\rangle U e_{j} \\
& =U\left(\sum_{j \in J}\left\langle U^{*} f, e_{j}\right\rangle e_{j}\right)=U\left(U^{*} f\right)
\end{aligned}
$$




$$
=U U^{*} f
$$

That is, the frame operator of the Bessel sequence $\left\{U e_{j}\right\}_{j \in J}$ is $U U^{*}$. Now, define

$$
\Theta: \operatorname{Bess}(\mathcal{H}) \rightarrow B(\mathcal{H}) \quad \text { by } \quad \Theta\left(\left\{f_{j}\right\}_{j \in J}\right)=U_{F}
$$

and

$$
\Omega: B(\mathcal{H}) \rightarrow \operatorname{Bess}(\mathcal{H}) \quad \text { by } \quad \Omega(U)=\left\{U e_{j}\right\}_{j \in J} .
$$

By the above mentioned argument, we see that $\Theta$ and $\Omega$ are well defined. Uniqueness of $U_{F}$ together with (2.1) implies that

$$
\Omega\left(\Theta\left(\left\{f_{j}\right\}_{j \in J}\right)\right)=\Omega\left(U_{F}\right)=\left\{U_{F} e_{j}\right\}_{j \in J}=\left\{f_{j}\right\}_{j \in J}
$$

and

$$
\Theta(\Omega(U))=\Theta\left(\left\{U e_{j}\right\}_{j \in J}\right)=U .
$$

This completes the proof.

For a Bessel sequence $F=\left\{f_{j}\right\}_{j \in J}$ in $\mathcal{H}$ we denote by $U_{F}$ the operator obtained in Theorem 3. As a consequence of Theorem 3 we have the following known result (see e.g. [1]).

Corollary 1. Let $\mathcal{H}$ be a Hilbert spaces with the Bessel sequence space $\operatorname{Bess}(\mathcal{H})$ and $\Theta$ be as in (2.3). Then,

$$
\operatorname{KFr}(\mathcal{H})=\Omega\{A \in B(\mathcal{H}): R(K) \subseteq R(A)\}
$$

and so

$$
\operatorname{Fr}(\mathcal{H})=\Omega\{A \in B(\mathcal{H}): A \text { is surjective }\} .
$$

Proof. It follows from Theorem 1 and (2.2) that a Bessel sequence $F=\left\{f_{j}\right\}_{j \in J}$ is $K$-frame if and only if there exists $\alpha>0$ such that $U_{F} U_{F}^{*}=S_{F} \geq \alpha K K^{*}$. By Douglas' factorization theorem (Theorem 2), it is equivalent to $R(K) \subseteq R\left(U_{F}\right)$. Moreover, taking $K=I$ (the identity operator) we obtain desired conclusion.

One-to-one correspondence in Theorem 3 motivate us to introduce the following notions.

Definition 5. Let $\left\{e_{j}\right\}_{j \in J}$ be an arbitrary orthonormal basis for $\mathcal{H}$ and $\left\{f_{j}\right\}_{j \in J}$ be a Bessel sequence in $\mathcal{H}$. Adjoint of $\left\{f_{j}\right\}_{j \in J}$ is a Bessel sequence $\left\{g_{j}\right\}_{j \in J}$ in $\mathcal{H}$ such that

$$
\sum_{j \in J}\left\langle f, g_{j}\right\rangle\left\langle e_{j}, g\right\rangle=\sum_{j \in J}\left\langle f_{j}, g\right\rangle\left\langle f, e_{j}\right\rangle, \quad \forall f, g \in \mathcal{H} .
$$

We denote by $\left\{f_{j}^{*}\right\}_{j \in J}$ the adjoint of $\left\{f_{j}\right\}_{j \in J}$. If

$$
\sum_{j \in J}\left\langle f, f_{j}\right\rangle\left\langle e_{j}, g\right\rangle=\sum_{j \in J}\left\langle f_{j}, g\right\rangle\left\langle f, e_{j}\right\rangle, \quad \forall f, g \in \mathcal{H},
$$

we say that $\left\{f_{j}\right\}_{j \in J}$ is self-adjoint. Also, $\left\{f_{j}\right\}_{j \in J}$ is positive if

$$
\sum_{j \in J}\left\langle f_{j}, f\right\rangle\left\langle f, e_{j}\right\rangle \geq 0, \quad \forall f \in \mathcal{H} .
$$


A positive frame is a frame in which inequality (2.6) holds.

Theorem 4. Let $\left\{f_{j}\right\}_{j \in J}$ be a Bessel sequence in $\mathcal{H}$. Then the followings hold:

(i) $\left\{f_{j}\right\}_{j \in J}$ has a unique adjoint in $\mathcal{H}$;

(ii) $\left\{f_{j}\right\}_{j \in J}$ is self-adjoint if and only if $U_{F}$ is self-adjoint;

(iii) $\left\{f_{j}\right\}_{j \in J}$ is positive if and only if $U_{F}$ is positive.

Proof. $(i)$ Let $\left\{f_{j}\right\}_{j \in J}$ be a Bessel sequence in $\mathcal{H}$. Consider the Bessel sequence $\left\{g_{j}\right\}_{j \in J}=\left\{U_{F}^{*} e_{j}\right\}_{j \in J}$. Now, we verify the equation (2.4). Let $f, g \in \mathcal{H}$. Then

$$
\begin{aligned}
\sum_{j \in J}\left\langle f, g_{j}\right\rangle\left\langle e_{j}, g\right\rangle & =\sum_{j \in J}\left\langle f, U_{F}^{*} e_{j}\right\rangle\left\langle e_{j}, g\right\rangle \\
& =\sum_{j \in J}\left\langle U_{F} f, e_{j}\right\rangle\left\langle e_{j}, g\right\rangle \\
& =\left\langle\sum_{j \in J}\left\langle U_{F} f, e_{j}\right\rangle e_{j}, g\right\rangle \\
& =\left\langle U_{F} f, g\right\rangle \\
& =\left\langle f, U_{F}^{*} g\right\rangle \\
& =\left\langle f, \sum_{j \in J}\left\langle U_{F}^{*} g, e_{j}\right\rangle e_{j}\right\rangle \\
& =\sum_{j \in J}\left\langle e_{j}, U_{F}^{*} g\right\rangle\left\langle f, e_{j}\right\rangle \\
& =\sum_{j \in J}\left\langle U_{F} e_{j}, g\right\rangle\left\langle f, e_{j}\right\rangle=\sum_{j \in J}\left\langle f_{j}, g\right\rangle\left\langle f, e_{j}\right\rangle,
\end{aligned}
$$

so $\left\{U_{F}^{*} e_{j}\right\}_{j \in J}$ is an adjoint of $F=\left\{f_{j}\right\}_{j \in J}$. Uniqueness follows from uniqueness of $U_{F}$ in Theorem 3 and its adjoint.

(ii) By a similar computation as in $(i)$, we get that

$$
\left\langle U_{F} f, g\right\rangle=\left\langle f, U_{F}^{*} g\right\rangle=\sum_{j \in J}\left\langle f_{j}, g\right\rangle\left\langle f, e_{j}\right\rangle, \quad \forall f, g \in \mathcal{H},
$$

and

$$
\left\langle U_{F}^{*} f, g\right\rangle=\sum_{j \in J}\left\langle f, f_{j}\right\rangle\left\langle e_{j}, g\right\rangle, \quad \forall f, g \in \mathcal{H},
$$

which proves $(i i)$.

It follows from (2.7) that

$$
\left\langle U_{F} f, f\right\rangle=\sum_{j \in J}\left\langle f_{j}, f\right\rangle\left\langle f, e_{j}\right\rangle, \quad \forall f \in \mathcal{H},
$$

which gives us (iii).

Corollary 2. Let $\left\{f_{j}\right\}_{j \in J}$ be a Bessel sequence in $\mathcal{H}$.

(i) If $\left\{f_{j}\right\}_{j \in J}$ is self-adjoint, then $\left\{U_{F} f_{j}\right\}_{j \in J}$ is positive. 
(ii) If $V \in B(\mathcal{H})$ is self-adjoint, then $\left\{V^{2} e_{j}\right\}_{j \in J}$ is positive.

(iii) If $\left\{f_{j}\right\}_{j \in J}$ is positive, then there exists a self-adjoint operator $V \in B(\mathcal{H})$ such that $\left\{f_{j}\right\}_{j \in J}=\left\{V^{2} e_{j}\right\}_{j \in J}$.

Proof. (i) We have

$$
U_{F} f_{j}=U_{F} U_{F} e_{j}=U_{F}^{2} e_{j} .
$$

By Theorem 4, $U_{F}$ is self-adjoint and so $U_{F}^{2}=U_{F} U_{F}^{*} \geq 0$. Again, it follows from Theorem 4 that $\left\{U_{F} f_{j}\right\}_{j \in J}$ is positive.

(ii) Taking $\left\{f_{j}\right\}_{j \in J}=\left\{V e_{j}\right\}_{j \in J}$, we have $U_{F}=V$. Since $V$ is self-adjoint, then $\left\{f_{j}\right\}_{j \in J}$ is self-adjoint. Hence using the previous part, $\left\{V^{2} e_{j}\right\}_{j \in J}=\left\{V f_{j}\right\}_{j \in J}$ is positive.

(iii) Since $\left\{f_{j}\right\}_{j \in J}$ is positive, it follows from Theorem 4 that $U_{F}$ is positive. Put $V=U_{F}^{\frac{1}{2}}$. Then, $\left\{f_{j}\right\}_{j \in J}=\left\{U_{F} e_{j}\right\}_{j \in J}=\left\{V^{2} e_{j}\right\}_{j \in J}$.

Lemma 1. Let $F=\left\{f_{j}\right\}_{j \in J}$ be a Bessel sequence with the frame operator $S_{F}$. If $\left\{f_{j}\right\}_{j \in J}$ is positive, then $U_{F}=S_{F}^{\frac{1}{2}}$.

Proof. It follows from (2.2) that

$$
S_{F}=U_{F} U_{F}^{*}=U_{F}^{2} .
$$

By the uniqueness of positive square root we obtain the desired result.

The following lemma provides a basic tool to define product of Bessel sequences.

Lemma 2. Let $F=\left\{f_{j}\right\}_{j \in J}$ and $G=\left\{g_{j}\right\}_{j \in J}$ be two Bessel sequences in $\mathcal{H}$. Then, there exists a unique Bessel sequence $\left\{h_{j}\right\}_{j \in J}$ in $\mathcal{H}$ such that

$$
\sum_{j \in J}\left\langle f, h_{j}\right\rangle e_{j}=\sum_{j \in J} \sum_{i \in J}\left\langle f, f_{i}\right\rangle\left\langle e_{i}, g_{j}\right\rangle e_{j}, \quad \forall f \in \mathcal{H} .
$$

Proof. Put $\left\{h_{j}\right\}_{j \in J}=\left\{U_{F} U_{G} e_{j}\right\}_{j \in J}$. For each $f \in \mathcal{H}$ we have

$$
\begin{aligned}
\sum_{j \in J}\left\langle f, h_{j}\right\rangle e_{j} & =\sum_{j \in J}\left\langle f, U_{F} U_{G} e_{j}\right\rangle e_{j}=\sum_{j \in J}\left\langle U_{F}^{*} f, g_{j}\right\rangle e_{j} \\
& =\sum_{j \in J}\left\langle\sum_{i \in J}\left\langle U_{F}^{*} f, e_{i}\right\rangle e_{i}, g_{j}\right\rangle e_{j} \\
& =\sum_{j \in J} \sum_{i \in J}\left\langle U_{F}^{*} f, e_{i}\right\rangle\left\langle e_{i}, g_{j}\right\rangle e_{j} \\
& =\sum_{j \in J} \sum_{i \in J}\left\langle f, U_{F} e_{i}\right\rangle\left\langle e_{i}, g_{j}\right\rangle e_{j} \\
& =\sum_{j \in J} \sum_{i \in J}\left\langle f, f_{i}\right\rangle\left\langle e_{i}, g_{j}\right\rangle e_{j} .
\end{aligned}
$$


To prove the uniqueness, let $H=\left\{h_{j}\right\}_{j \in J}$ and $M=\left\{m_{j}\right\}_{j \in J}$ be two Bessel sequences in $\mathcal{H}$ for which the equality (2.8) holds. Hence,

$$
\sum_{j \in J}\left\langle f, h_{j}\right\rangle e_{j}=\sum_{j \in J} \sum_{i \in J}\left\langle f, f_{i}\right\rangle\left\langle e_{i}, g_{j}\right\rangle e_{j}=\sum_{j \in J}\left\langle f, m_{j}\right\rangle e_{j}, \quad \forall f \in \mathcal{H} .
$$

On the other hand, we have

$$
U_{H}^{*} f=\sum_{j \in J}\left\langle U_{H}^{*} f, e_{j}\right\rangle e_{j}=\sum_{j \in J}\left\langle f, U_{H} e_{j}\right\rangle e_{j}=\sum_{j \in J}\left\langle f, h_{j}\right\rangle e_{j}, \quad \forall f \in \mathcal{H} .
$$

Considering (2.9) and (2.10) we see that $U_{H}=U_{M}$ and so $\left\{h_{j}\right\}_{j \in J}=\left\{m_{j}\right\}_{j \in J \text {. }}$

For the Bessel sequences $\left\{f_{j}\right\}_{j \in J}$ and $\left\{g_{j}\right\}_{j \in J}$ in $\mathcal{H}$, we define product of them as the Bessel sequence obtained in Lemma 2.

Remark 2. By using the same argument as Theorem 4 and Lemma 2, we see that for the Bessel sequence $F=\left\{f_{j}\right\}_{j \in J}$ in $\mathcal{H}$, the operator $U_{F}$ is normal if and only if

$$
\sum_{j \in J} \sum_{i \in J}\left\langle f, f_{i}\right\rangle\left\langle e_{i}, f_{j}^{*}\right\rangle e_{j}=\sum_{j \in J} \sum_{i \in J}\left\langle f, f_{i}^{*}\right\rangle\left\langle e_{i}, f_{j}\right\rangle e_{j}, \quad \forall f \in \mathcal{H} .
$$

In this case we say that the Bessel sequence $\left\{f_{j}\right\}_{j \in J}$ is normal.

Theorem 5. The set Bess $(\mathcal{H})$ is an algebra with the following operations:

$$
\begin{aligned}
\left\{f_{j}\right\}_{j \in J}+\left\{g_{j}\right\}_{j \in J} & :=\left\{f_{j}+g_{j}\right\}_{j \in J}=\left\{\left(U_{F}+U_{G}\right) e_{j}\right\}_{j \in J}, \\
\alpha\left\{f_{j}\right\}_{j \in J} & :=\left\{\alpha f_{j}\right\}_{j \in J}=\left\{\left(\alpha U_{F}\right) e_{j}\right\}_{j \in J} \\
\left\{f_{j}\right\}_{j \in J} \cdot\left\{g_{j}\right\}_{j \in J} & =\left\{\left(U_{F} U_{G}\right) e_{j}\right\}_{j \in J} .
\end{aligned}
$$

The proof is straightforward.

Theorem 6. The function

$$
\Theta: \operatorname{Bess}(\mathcal{H}) \rightarrow B(\mathcal{H}) \quad \text { defined by } \quad \Theta\left(\left\{f_{j}\right\}_{j \in J}\right)=U_{F}
$$

is an algebra positive homomorphism.

\section{MEANS OF FRAMES}

Recall that power mean of two positive operators $A$ and $B$ is defined by

$$
\left(\frac{A^{r}+B^{r}}{2}\right)^{\frac{1}{r}} \quad r \in \mathbb{R} \backslash\{0\} .
$$

We begin with Arithmetic mean of two positive frames.

Theorem 7. Frame operator of Arithmetic mean of two positive frames is power mean of their frame operators for $r=\frac{1}{2}$. 
Proof. Let $F=\left\{f_{j}\right\}_{j \in J}$ and $G=\left\{g_{j}\right\}_{j \in J}$ be two positive frames in $\mathcal{H}$ with the frame operators $S_{F}$ and $S_{G}$, respectively. We know from Lemma 1 that

$$
f_{j}=U_{F} e_{j}=S_{F}^{\frac{1}{2}} e_{j}, \quad g_{j}=U_{G} e_{j}=S_{G}^{\frac{1}{2}} e_{j} .
$$

Since Arithmetic mean of $F=\left\{f_{j}\right\}_{j \in J}$ and $G=\left\{g_{j}\right\}_{j \in J}$ is

$$
\left\{\frac{f_{j}+g_{j}}{2}\right\}_{j \in J}=\left\{\left(\frac{U_{F}+U_{G}}{2}\right) e_{j}\right\}_{j \in J},
$$

considering (2.2), its frame operator is

$$
\left(\frac{U_{F}+U_{G}}{2}\right)\left(\frac{U_{F}+U_{G}}{2}\right)^{*}=\left(\frac{S_{F}^{1 / 2}+S_{G}^{1 / 2}}{2}\right)^{2}
$$

To extend the idea to other means we need apply functional calculus on $\operatorname{Bess}(\mathcal{H})$. Let $A$ be a normal operator on $\mathcal{H}, C(S p(A))$ be the set of all continuous functions on the spectrum of $A$ and $C^{*}(A, I)$ be the abalian $C^{*}$-algebra generated by $A$ and $I$. An immediate consequence of Gelfand's Theorem [10, Theorem 2.1.10] is that there is an isometric $*$-isomorphism between $C(S p(A))$ and $C^{*}(A, I)$. Hence, for any $\varphi \in$ $C(S p(A))$ the operator $\varphi(A)$ is in $C^{*}(A, I)$. Moreover, spectral mapping theorem [10, Theorem 2.1.14] guaranties that $\varphi(S p(A))=S p(\varphi(A))$. So, if $\varphi(S p(A)) \subseteq[0,+\infty)$, then $\varphi(A)$ is a positive operator.

Now, we define new Bessel sequences by using functional calculus. Let $F=$ $\left\{f_{j}\right\}_{j \in J}$ be a Bessel sequence in $\mathcal{H}$ for which $U_{F}$ is normal operator and $\varphi \in C\left(S p\left(U_{F}\right)\right)$. We define

$$
\varphi(F)=\left\{\varphi\left(U_{F}\right) e_{j}\right\}_{j \in J} .
$$

For example, if the Bessel sequence $F=\left\{f_{j}\right\}_{j \in J}$ is positive, then $\varphi(t)=t^{\frac{1}{2}}$ is continuous on $\operatorname{Sp}\left(U_{F}\right)$ and we have

$$
\varphi(F)=\left\{\varphi\left(U_{F}\right) e_{j}\right\}_{j \in J}=\left\{U_{F}^{\frac{1}{2}} e_{j}\right\}_{j \in J} .
$$

We denote this new Bessel sequences by $\left\{f_{j}\right\}_{j \in J}^{\frac{1}{2}}$.

Let $\mathcal{H}$ and $\mathcal{K}$ be two Hilbert spaces. Recall that a linear mapping $\Phi: B(\mathcal{H}) \rightarrow B(\mathcal{K})$ is called positive map if $\Phi$ maps positive elements to positive elements.

Definition 6. Let $\mathcal{H}$ and $\mathcal{K}$ be two separable Hilbert spaces. Every positive linear maps $\Phi: B(\mathcal{H}) \rightarrow B(\mathcal{K})$ induces positive linear map $\widehat{\Phi}: \operatorname{Bess}(\mathcal{H}) \rightarrow \operatorname{Bess}(\mathcal{K})$ such that

$$
\widehat{\Phi}(F)=\left\{\Phi\left(U_{F}\right) \widehat{e}_{j}\right\}_{j \in J},
$$

where $F=\left\{f_{j}\right\}_{j \in J}$ is a Bessel sequences in $\mathcal{H}$ and $\left\{\widehat{\boldsymbol{e}}_{j}\right\}_{j \in J}$ is an arbitrary and fixed orthonormal bases for $\mathcal{K}$. 
Lemma 3. Let $\mathcal{H}$ and $\mathcal{K}$ be two separable Hilbert spaces with the Bessel sequence spaces Bess $(\mathcal{H})$ and Bess $(\mathcal{K})$, respectively. For each positive linear map $\Phi: B(\mathcal{H}) \rightarrow B(\mathcal{K})$, the following diagram commutes:

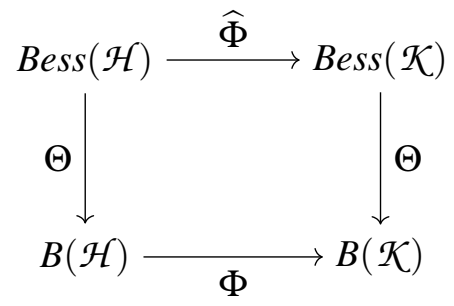

where $\Theta$ is defined by (2.3).

Proof. Let $F=\left\{f_{j}\right\}_{j \in J}$ be a Bessel sequence in $\mathcal{H}$. Considering (2.3) we have $\Phi\left(\Theta\left\{f_{j}\right\}_{j \in J}\right)=\Phi\left(U_{F}\right)$. On the other hand,

$$
\Theta\left(\widehat{\Phi}\left\{f_{j}\right\}_{j \in J}\right)=\Theta\left(\Phi\left(U_{F}\right) \widehat{e}_{j}\right)=\Phi\left(U_{F}\right) .
$$

Let $B_{+}(\mathcal{H})$ be the set of all positive operators of $B(\mathcal{H})$ and $P_{N}[B(\mathcal{H}), B(\mathcal{K})]$ be the set of all normalized positive linear maps from $B(\mathcal{H})$ to $B(\mathcal{K})$. Micic and Pecaric in [9] introduced the following weighted power means of positive operators and studied related inequalities. Let $\Phi_{j} \in P_{N}[B(\mathcal{H}), B(\mathcal{K})], A_{i} \in B_{+}(\mathcal{H})$ with $\operatorname{Sp}\left(A_{i}\right) \subseteq[m, M]$ for some scalars $0<m<M$ and $\omega_{i} \in \mathbb{R}^{+}$such that $\sum_{i=1}^{k} \omega_{i}=1(i=1, \ldots, k)$. Define

$$
M_{k}^{[r]}(\mathrm{A}, \Phi, \omega):=\left(\sum_{i=1}^{k} \omega_{i} \Phi_{i}\left(A_{i}^{r}\right)\right)^{1 / r} \quad \text { if } \quad r \in \mathbb{R} \backslash\{0\},
$$

where $A=\left(A_{1}, \ldots, A_{k}\right), \Phi=\left(\Phi_{1}, \ldots, \Phi_{k}\right)$ and $\omega=\left(\omega_{1}, \ldots, \omega_{k}\right)$. Similarly, if $F_{i}=$ $\left\{f_{i j}\right\}_{j \in J}$ are positive frames in $\mathcal{H}$, we define their weighted power means as

$$
\mathcal{M}_{k}^{[r]}(\mathrm{F}, \Phi, \omega):=\left(\sum_{i=1}^{k} \omega_{i} \widehat{\Phi}_{i}\left(\left\{f_{i j}\right\}_{j \in J}^{r}\right)\right)^{1 / r} \quad \text { if } \quad r \in \mathbb{R} \backslash\{0\},
$$

where $F=\left(F_{1}, \ldots, F_{k}\right)$.

Theorem 8. Frame operator of weighted power mean of positive frames for $r$ is weighted power mean of their frame operators for $\frac{r}{2}$.

Proof. Let $\left\{e_{j}\right\}_{j \in J}$ and $\left\{\widehat{e}_{j}\right\}_{j \in J}$ be arbitrary and fixed orthonormal bases for $\mathcal{H}$ and $\mathcal{K}$, respectively. Let $F_{i}=\left\{f_{i j}\right\}_{j \in J}(i=1, \ldots, k)$ be positive frames in $\mathcal{H}$ with the frame operators $S_{F_{i}}$ 's. The weighted power mean of the positive frames $F_{i}=\left\{f_{i j}\right\}_{j \in J}$ 
is a positive frame in $\mathcal{K}$ such that

$$
\begin{aligned}
\mathcal{M}_{k}^{[r]}(\mathrm{F}, \Phi, \omega) & =\left(\sum_{i=1}^{k} \omega_{i} \widehat{\Phi}_{i}\left(\left\{f_{i j}\right\}_{j \in J}^{r}\right)\right)^{1 / r} \\
& =\left(\sum_{i=1}^{k} \omega_{i} \Phi_{i}\left(U_{F_{i}}^{r}\right)\right)^{1 / r} \widehat{e}_{j}=\left(\sum_{i=1}^{k} \omega_{i} \Phi_{i}\left(S_{F_{i}}^{\frac{r}{2}}\right)\right)^{1 / r} \widehat{e}_{j}:=V \widehat{e}_{j} .
\end{aligned}
$$

Therefore, the frame operator of $\mathcal{M}_{k}^{[r]}(\mathrm{F}, \Phi, \omega)$ is

$$
\begin{aligned}
V V^{*} & =\left(\sum_{i=1}^{k} \omega_{i} \Phi_{i}\left(S_{F_{i}}^{\frac{r}{2}}\right)\right)^{1 / r}\left(\sum_{i=1}^{k} \omega_{i} \Phi_{i}\left(S_{F_{i}}^{\frac{r}{2}}\right)\right)^{1 / r} \\
& =\left(\sum_{i=1}^{k} \omega_{i} \Phi_{i}\left(S_{F_{i}}^{\frac{r}{2}}\right)\right)^{2 / r}=M_{k}^{\left[\frac{r}{2}\right]}\left(S_{F}, \Phi, \omega\right),
\end{aligned}
$$

where $S_{F}=\left(S_{F_{1}}, \ldots, S_{F_{k}}\right)$.

\section{ACKNOWLEDGEMENT}

We gratefully thank to the reviewers due to their helpful comments for improving paper.

\section{REFERENCES}

[1] O. Christensen, An introduction to frames and Riesz bases. , 2nd ed. Basel: Birkhäuser/Springer, 2016. doi: 10.1007/978-3-319-25613-9.

[2] I. Daubechies, A. Grossmann, and Y. Meyer, "Painless nonorthogonal expansions." J. Math. Phys., vol. 27, pp. 1271-1283, 1986, doi: 10.1007/978-3-319-25613-9.

[3] M. Ding, X. Xiao, and X. Zeng, "Tight $K$-frames in Hilbert spaces." Acta Math. Sin., Chin. Ser., vol. 56, no. 1, pp. 105-112, 2013.

[4] R. G. Douglas, "On majorization, factorization, and range inclusion of operators on Hilbert space." Proc. Am. Math. Soc., vol. 17, pp. 413-415, 1966, doi: 10.2307/2035178.

[5] R. J. Duffin and A. C. Schaeffer, "A class of nonharmonic Fourier series." Trans. Am. Math. Soc., vol. 72, pp. 341-366, 1952, doi: 10.2307/1990760.

[6] M. H. Faroughi and R. Ahmadi, "Some properties of $C$-fusion frames." Turk. J. Math., vol. 34, no. 3, pp. 393-416, 2010.

[7] M. Fornasier and H. Rauhut, "Continuous frames, function spaces, and the discretization problem.” J. Fourier Anal. Appl., vol. 11, no. 3, pp. 245-287, 2005, doi: 10.1007/s00041-005-4053-6.

[8] L. Găvruţa, "Frames for operators." Appl. Comput. Harmon. Anal., vol. 32, no. 1, pp. 139-144, 2012, doi: 10.1016/j.acha.2011.07.006.

[9] J. Mićić and J. Pečarić, "Order among power means of positive operators.” Sci. Math. Jpn., vol. 61, no. 1, pp. 25-46, 2005.

[10] G. J. Murphy, $C^{*}$-algebras and operator theory. Boston, MA etc.: Academic Press, Inc., 1990.

[11] S. Obeidat, S. Samarah, P. G. Casazza, and J. C. Tremain, "Sums of Hilbert space frames." J. Math. Anal. Appl., vol. 351, no. 2, pp. 579-585, 2009, doi: 10.1016/j.jmaa.2008.10.040. 
[12] W. Sun, " $g$-frames and $g$-Riesz bases." J. Math. Anal. Appl., vol. 322, no. 1, pp. 437-452, 2006, doi: 10.1016/j.jmaa.2005.09.039.

[13] X. Xiao, Y. Zhu, and L. Găvruța, "Some properties of $K$-frames in Hilbert spaces." Result. Math., vol. 63, no. 3-4, pp. 1243-1255, 2013, doi: 10.1007/s00025-012-0266-6.

Authors' addresses

Esmaeil Alizadeh

Department of Mathematics, Marand Branch, Islamic Azad University, Marand, Iran

E-mail address: e_alizadeh@marandiau.ac.ir

Asghar Rahimi

Department of Mathematics, University of Maragheh, Maragheh, Iran

E-mail address: rahimi@maragheh.ac.ir

Mortaza Rahmani

Young Researchers and Elite Club, Ilkhchi Branch, Islamic Azad University, Ilkhchi, Iran

E-mail address: m_rahmani26@yahoo.com 